\title{
Osimertinib for the treatment of epidermal growth factor receptor- mutated non-small cell lung cancer patients with leptomeningeal metastases and different T790M status
}

\author{
Haiyan Xu ${ }^{1 \#}$, Hengqi Chen ${ }^{2 \#}$, Jianxin Kong ${ }^{3}$, Ye Zhang ${ }^{4}$, Shan Liu ${ }^{1}$, Guangjian Yang ${ }^{5}$, Lu Yang ${ }^{5}$, \\ Yan Wang $^{5 \wedge}$
}

${ }^{1}$ Department of Comprehensive Oncology, National Cancer Center/National Clinical Research Center for Cancer/Cancer Hospital, Chinese Academy of Medical Sciences and Peking Union Medical College, Beijing, China; ${ }^{2}$ Thoracic Surgery Department, National Cancer Center/ National Clinical Research Center for Cancer/Cancer Hospital, Chinese Academy of Medical Sciences and Peking Union Medical College, Beijing, China; ${ }^{3}$ Department of Neurosurgery, National Cancer Center/National Clinical Research Center for Cancer/Cancer Hospital, Chinese Academy of Medical Sciences and Peking Union Medical College, Beijing, China; ${ }^{4}$ Department of Radiation Oncology, National Cancer Center/National Clinical Research Center for Cancer/Cancer Hospital, Chinese Academy of Medical Sciences and Peking Union Medical College, Beijing, China; ${ }^{5}$ Department of Medical Oncology, National Cancer Center/National Clinical Research Center for Cancer/Cancer Hospital, Chinese Academy of Medical Sciences and Peking Union Medical College, Beijing, China

Contributions: (I) Conception and design: H Xu, J Kong, Y Zhang, Y Wang; (II) Administrative support: G Yang; (III) Provision of study materials or patients: H Chen, J Kong, Y Wang; (IV) Collection and assembly of data: S Liu, G Yang, L Yang; (V) Data analysis and interpretation: S Liu, G Yang, L Yang; (VI) Manuscript writing: All authors; (VII) Final approval of manuscript: All authors.

\#These authors contributed equally to this work.

Correspondence to: Yan Wang. Department of Medical Oncology, National Cancer Center/National Clinical Research Center for Cancer/Cancer Hospital, Chinese Academy of Medical Sciences and Peking Union Medical College, No. 17 Panjiayuan Street South, Chaoyang District, Beijing, China. Email: wangyanyifu@163.com.

Background: Leptomeningeal metastasis (LM) is a catastrophic complication for patients with non-small cell lung cancer (NSCLC) and carries an extremely poor prognosis. The efficacy of osimertinib $80 \mathrm{mg}$ once daily for epidermal growth factor receptor-mutated (EGFRm) NSCLC with LM has yet to be fully assessed. This study aimed to investigate the efficacy of osimertinib in such patients and their genetic profiles at the time of LM diagnosis.

Methods: From January 2016 to April 2020, pretreated EGFRm NSCLC patients who had progressed with cytologically confirmed symptomatic LM and received osimertinib $80 \mathrm{mg}$ once daily were enrolled retrospectively. The objective response rate (ORR) and disease control rate (DCR) were evaluated, along with progression-free survival (PFS) and overall survival (OS). Next-generation sequencing of paired samples of cerebrospinal fluid and plasma collected at LM diagnosis was performed simultaneously.

Results: Forty cases of EGFRm lung adenocarcinoma with LM were analyzed. Females accounted for $75.0 \%$ of enrollees. Of the patients, $37.5 \%$ had a poor Eastern Cooperative Oncology Group score $(\geq 2)$. Twelve patients had received at least 2 prior lines of treatment. All patients received osimertinib treatment regardless of their T790M status. According to the Response Assessment in Neuro-Oncology (RANO)LM criteria, the ORR and DCR were $20.0 \%$ and $95.0 \%$, respectively. The median PFS and OS were 10.0 (95\% CI: 7.7-12.3) and 15.1 months (95\% CI: 11.0-19.4), respectively. No significant difference was observed between T790M-negative patients ( $n=24)$ and T790M-positive patients $(n=16)$ with respect to PFS [median, 10.8 (95\% CI: 7.7-13.8) vs. 8.8 months (95\% CI: 7.3-10.3), HR=0.595, P=0.158] or OS [median, 17.2 (95\% CI: 8.7-25.7) vs. 11.4 months (95\% CI: 3.9-19.0), HR=0.913, $\mathrm{P}=0.822]$. The detection rate of

^ ORCID: Haiyan Xu, 0000-0002-0048-3191; Lu Yang, 0000-0002-4286-9586; Yan Wang, 0000-0002-1743-6383. 
EGFR sensitizing mutations in cerebrospinal fluid was higher than that in plasma $(97.5 \%$ s. $50 \%, \mathrm{P}=0.311)$, whereas the incidence of T790M detection in cerebrospinal fluid was significantly lower than that in plasma (20.0\% vs. $32.5 \%, \mathrm{P}=0.043)$.

Conclusions: Osimertinib $80 \mathrm{mg}$ once daily shows good efficacy in pretreated EGFRm NSCLC patients with LM regardless of their T790M status. Combining cerebrospinal fluid and plasma testing can aid in revealing more genetic information.

Keywords: Non-small cell lung cancer (NSCLC); EGFR; leptomeningeal metastasis (LM); cerebrospinal fluid; osimertinib

Submitted Feb 23, 2021. Accepted for publication Apr 09, 2021.

doi: 10.21037/atm-21-1249

View this article at: http://dx.doi.org/10.21037/atm-21-1249

\section{Introduction}

Leptomeningeal metastasis (LM) is a catastrophic complication of non-small cell lung cancer (NSCLC). Of patients with epidermal growth factor receptor-mutated (EGFRm) NSCLC, approximately $25 \%$ have central nervous system (CNS) metastasis at the time of their initial diagnosis of advanced disease, and $34.2 \%$ to $52.9 \%$ develop brain metastases during treatment (1). Furthermore, EGFRm NSCLC is associated with a higher prevalence of LM than wild-type EGFR NSCLC (9.4\% vs. $1.7 \%)$ (2), with almost $10 \%$ of patients with EGFRm NSCLC eventually developing LM. The median time from advanced NSCLC diagnosis to LM is 13.6 months (3). Patients with LM experience severe clinical symptoms and have a poor prognosis, with a median overall survival (OS) of only 3 to 11 months $(4,5)$.

Before the emergence of $E G F R$ tyrosine kinase inhibitors (EGFR-TKIs), traditional treatment strategies for LM included whole-brain radiotherapy, and systemic and intrathecal chemotherapy. Despite whole-brain radiotherapy serving as a treatment option for patients with LM, it has not been reported to attain therapeutic benefit in terms of improving OS (6). In a previously published analysis, intrathecal chemotherapy proved to be a fairly effective treatment option for NSCLC patients with LM and could improve neurological symptoms (7); however, there is still no consensus for the dose selection or treatment duration of intrathecal therapy. Currently, targeted therapies based on genetic alterations are recommended as first-line standard treatment for patients with advanced NSCLC harboring sensitive EGFR gene mutations (8-13). Several studies have also evidenced that the administration of EGFR-TKIs before or beyond LM diagnosis can prolong the OS of patients with EGFRm NSCLC $(14,15)$.

Osimertinib is an irreversible third generation EGFRTKI with a high level of CNS penetration that inhibits both EGFR sensitive mutations and resistant EGFR-T790M mutations. In the AURA3 study, osimertinib showed promising activity in patients with EGFRm and T790Mpositive advanced NSCLC who progressed after previous treatment with EGFR-TKIs, achieving a median CNS PFS of 11.7 months (16). In the phase I BLOOM study, the efficacy of osimertinib $160 \mathrm{mg}$ once daily attained a median OS of 11.0 months in patients with LM (17). However, the efficacy of osimertinib $80 \mathrm{mg}$ once daily in patients with EGFRm NSCLC with LM based on T790M status has yet to be fully assessed in clinical practice.

Therefore, the present study sought to investigate the efficacy of osimertinib in pretreated patients with LM according to their T790M status, and to compare the genetic profiles of paired cerebrospinal fluid (CSF) and plasma samples collected from the patients at the time of LM diagnosis. It is hoped that this study will deepen the understanding of the characteristics of patients with LM who acquire resistance to first-generation TKIs. We present the following article in accordance with the STROBE reporting checklist (available at http://dx.doi.org/10.21037/ atm-21-1249).

\section{Methods}

\section{Patients and samples}

From January 2016 to April 2020, patients with EGFRm NSCLC who had progressed with cytologically confirmed symptomatic LM during first-generation EGFR-TKI therapy and had received osimertinib $80 \mathrm{mg}$ once daily until 
either radiographically progressive disease or unacceptable drug-related toxicity occurred were retrospectively enrolled from the National Cancer Center/Cancer Hospital, Chinese Academy of Medical Sciences. As an observational study without therapeutic intervention, the present study was exempted from obtaining patient informed consent, and the study was approved by the institutional ethics committee of National Cancer/Cancer Hospital, Chinese Academy of Medical Sciences \& Peking Union Medical College (approval 20-114/2310). The study was conducted in accordance with the Declaration of Helsinki (as revised in 2013).

All patients with symptomatic LM who met the following criteria were eligible: (I) aged $\geq 18$ years; (II) confirmed with metastatic lung adenocarcinoma with $E G F R$ sensitizing mutation and pretreated with firstgeneration EGFR-TKIs; (III) LM confirmed by positive CSF cytological examination; (IV) at least 1 measured lesion that could be evaluated repeatedly by magnetic resonance imaging (MRI) or computed tomography (CT); and (V) an Eastern Cooperative Oncology Group performance status (ECOG PS) score of $\leq 3$. EGFR status was retrospectively determined by real-time polymerase chain reaction (PCR) analysis or next-generation sequencing (NGS) of tumor tissues obtained from the primary or metastatic sites. CSF was collected at the time of LM diagnosis by lumbar puncture for cytological examination, and routine and biochemical testing. Genomic DNA was also extracted from $10 \mathrm{ml}$ of CSF or plasma at baseline by hybrid capture-based NGS testing.

Patients were excluded if they had two or more driver mutations simultaneously such as ALK, KRAS, MET application, HER-2 insertion, ROS1 and RET fusion other than $E G F R$ mutation, and received osimertinib treatment for less than 7 days.

\section{Efficacy evaluation and definitions}

CT examination of the neck, chest, and abdomen, brain MRI, and or whole-body bone scans were performed for imaging evaluation. Radiologic assessment was conducted at baseline, and then at approximately 2-month intervals. Intracranial response was assessed by investigators according to the Response Assessment in Neuro-Oncology (RANO)-LM radiologic criteria (18). Overall systemic response was defined by the Response Evaluation Criteria in Solid Tumors (RECIST) version 1.1 (19). The objective response rate (ORR) was defined as the percentage of patients who showed a complete response (CR) or partial response (PR). The disease control rate (DCR) was defined as the percentage of patients who were evaluated as CR, $\mathrm{PR}$, or stable disease (SD). PFS was defined as the time from the $1^{\text {st }}$ day of osimertinib initiation to the time of disease progression or death. OS was calculated from the $1^{\text {st }}$ day date of osimertinib treatment after the diagnosis of LM until death or the last follow-up (April 1, 2020). Patients' smoking history and ECOG PS data were collected from their electronic medical records, along with their clinical information and survival outcome.

\section{Statistical analysis}

Statistical analyses were carried out with SPSS version 16.0 (SPSS Inc., Chicago, IL, USA). Patient and treatment characteristics were presented as descriptive statistics, as appropriate. Data for dichotomous variables were presented as a percentage. The Kaplan-Meier method was used to estimate PFS and OS, and the log-rank test was used to compare difference between subgroups, as well as hazard ratios (HRs) and $95 \%$ confidence intervals (CIs) was estimated using a Cox model. A two-tailed test with $\mathrm{P}<0.05$ was considered to be statistically significant. Survival curves were generated with GraphPad Prism version 5.0 (GraphPad Software, San Diego, CA). The sensitivity of EGFR gene mutation was calculated in CSF and in plasma, respectively.

\section{Results}

\section{Baseline characteristics of patients with LM}

Forty cases of EGFRm adenocarcinoma with symptomatic LM were enrolled in this analysis. The study flow chart is shown in supplementary Appendix (Figure S1). Baseline characteristics of the study participants are summarized in Table 1. The study participants had a median age of 56 years (range: 35-69 years), and the majority of them were female $(75.0 \%)$. Of the enrolled patients, $37.5 \%(15 / 40)$ had a poor ECOG score ( $\geq 2)$, and $77.5 \%$ (31/40) were nonsmokers. At the time of LM diagnosis, coexisting brain metastases were reported in $87.5 \%$ of the cases. EGFR mutations were detected in exon 19 deletion $(\mathrm{n}=15)$, exon $21 \mathrm{~L} 858 \mathrm{R}$ mutation $(\mathrm{n}=23)$, and compound mutations $(\mathrm{n}=2)$, including one with exon $21 \mathrm{~L} 858 \mathrm{R}$ plus exon 20 S768I and another with exon $21 \mathrm{~L} 861 \mathrm{R}$ plus exon $21 \mathrm{~L} 833 \mathrm{~F}$. More than half of the patients (57.5\%) with LM had mutationsin 
Table 1 Baseline characteristics in EGFRm NSCLC with LM

\begin{tabular}{|c|c|}
\hline Characteristics & $\mathrm{n}=40$ \\
\hline \multicolumn{2}{|l|}{ Age, n (\%) } \\
\hline$\geq 60$ years & $19(47.5)$ \\
\hline$<60$ years & $21(52.5)$ \\
\hline \multicolumn{2}{|l|}{ Gender, n (\%) } \\
\hline Male & $10(25.0)$ \\
\hline Female & $30(75.0)$ \\
\hline \multicolumn{2}{|l|}{ Smoking history, n (\%) } \\
\hline Former/current & $9(22.5)$ \\
\hline Never & $31(77.5)$ \\
\hline \multicolumn{2}{|l|}{ Histology, n (\%) } \\
\hline Adenocarcinoma & $40(100.0)$ \\
\hline \multicolumn{2}{|l|}{ ECOG score, n (\%) } \\
\hline $0-1$ & $25(62.5)$ \\
\hline$\geq 2$ & $15(37.5)$ \\
\hline \multicolumn{2}{|c|}{$\begin{array}{l}\text { Coexisting main metastatic sites besides } \\
\text { LM, } \mathrm{n}(\%)\end{array}$} \\
\hline Brain & $35(87.5)$ \\
\hline Bone & $22(55.0)$ \\
\hline Lung & $16(40.0)$ \\
\hline Lymph nodes & $15(37.5)$ \\
\hline Others & $5(12.5)$ \\
\hline \multicolumn{2}{|c|}{ Primary EGFR-sensitive mutations, n (\%) } \\
\hline 19 deletion & $15(37.5)$ \\
\hline $21 \mathrm{~L} 858 \mathrm{R}$ & $23(57.5)$ \\
\hline Compound mutations ${ }^{\ddagger}$ & $2(5.0)$ \\
\hline \multicolumn{2}{|c|}{ Prior EGFR-TKIs treatment, n (\%) } \\
\hline Gefitinib & $12(30.0)$ \\
\hline Erlotinib & $15(37.5)$ \\
\hline Icotinib & $13(32.5)$ \\
\hline \multicolumn{2}{|l|}{ Prior chemotherapy, n (\%) } \\
\hline Yes & $12(30.0)$ \\
\hline No & $6(15.0)$ \\
\hline \multicolumn{2}{|c|}{ Whole-brain radiotherapy, n (\%) } \\
\hline Yes & $15(37.5)$ \\
\hline No & $25(62.5)$ \\
\hline \multicolumn{2}{|l|}{ Intrathecal treatment, $\mathrm{n}(\%)$} \\
\hline$>4$ times & $26(65.0)$ \\
\hline$\leq 4$ times & $14(35.0)$ \\
\hline
\end{tabular}

; , one patient had exon $21 \mathrm{~L} 858 \mathrm{R}$ and exon $20 \mathrm{~S} 768 \mathrm{I}$, and another had exon $21 \mathrm{~L} 861 \mathrm{R}$ and exon 21 L833F. EGFRm, epidermal growth factor receptor-mutated; NSCLC, non-small cell lung cancer; LM, leptomeningeal metastasis; ECOG, Eastern Cooperative Oncology Group.
EGFR exon $21 \mathrm{~L} 858 \mathrm{R}$. All of the patients received firstgeneration EGFR-TKI therapy, and 12 (30\%) patients had received at least 2 prior lines of treatment. All patients had received intrathecal injection of methotrexate treatment and 26 patients $(65 \%)$ had received intrathecal methotrexate treatment more than 4 times. Fifteen patients $(37.5 \%)$ had received brain radiotherapy.

\section{Therapeutic response and survival analysis of patients with EGFRm NSCLC and LM based on T790M status}

For the 40 patients with EGFRm NSCLC and LM, no patients discontinued or reduced osimertinib $80 \mathrm{mg}$ treatment due to aside effect. The median time from diagnosis of advanced NSCLC to LM was 18.6 months (95\% CI: 14.4-22.7). With respect to intracranial efficacy, none of the patients achieved CR, while PR, SD, and PD were achieved in 8, 30, and 2 cases, respectively. According to the RANO-LM criteria, the intracranial ORR and DCR were $20.0 \%$ and $95.0 \%$, respectively. The extracranial ORR and DCR were $40.0 \%$ and $100 \%$ (Table 2).

At the date of data cutoff (April 1, 2020), 30 (75.0\%) patients had died. The median follow-up time from diagnosis of LM to data cutoff was 34.5 months (range: $2.4-$ 36.9 months). The median PFS and OS were 10.0 months (95\% CI: 7.7-12.3) and 15.1 months (95\% CI: 11.0-19.4), respectively (Figure 1). To further determine the efficacy of osimertinib with respect to different T790M status, the patients were divided into 2 groups based on their T790M status. Positive T790M mutation status was confirmed by a positive result in either CSF or plasma testing. Osimertinib yielded a similar PFS in T790M-negative patients $(\mathrm{n}=24)$ and T790M-positive patients ( $\mathrm{n}=16)$ with $\mathrm{LM}$, with the median PFS being 10.8 months (95\% CI: 7.7-13.8) and 8.8 months (95\% CI: 7.3-10.3), respectively (HR 0.595, 95\% CI: $0.287-1.233, \mathrm{P}=0.158)$. The T790M-negative and T790M-positive groups had a median OS of 17.2 months (95\% CI: 8.7-25.7) and 11.4 months (95\% CI: 3.9-19.0), respectively (HR 0.913, 95\% CI: 0.423-1.982, $\mathrm{P}=0.822)$. No significant difference was observed in PFS or OS in either of the groups (Figure 2). Consistent results were observed when T790M status was detected in plasma (median PFS, 11.1 months in T790M-negative patients vs. 8.6 months in T790M-positive patients, $\mathrm{P}=0.163$ ) and in CSF (median PFS, 10.2 months in T790M-negative patients vs. 8.6 months in T790M-positive patients, $\mathrm{P}=0.076$, Figure S2). 
Table 2 Efficacy evaluation of osimertinib

\begin{tabular}{lcr}
\hline The best response & Intra-cranial (\%) & Extra-cranial (\%) \\
\hline CR & 0 & 0 \\
PR & $8(20.0)$ & $16(40.0)$ \\
SD & $30(75.0)$ & $38(60.0)$ \\
PD & $2(5.0)$ & 0 \\
ORR (CR + PR) & $8(20.0)$ & $16(40.0)$ \\
DCR (CR + PR + SD) & $38(95.0)$ & $100(100.0)$ \\
\hline
\end{tabular}

$\mathrm{CR}$, complete response; PR, partial response; SD, stable disease; PD, progressive disease; ORR, objective response rate; $\mathrm{DCR}$, disease control rate.

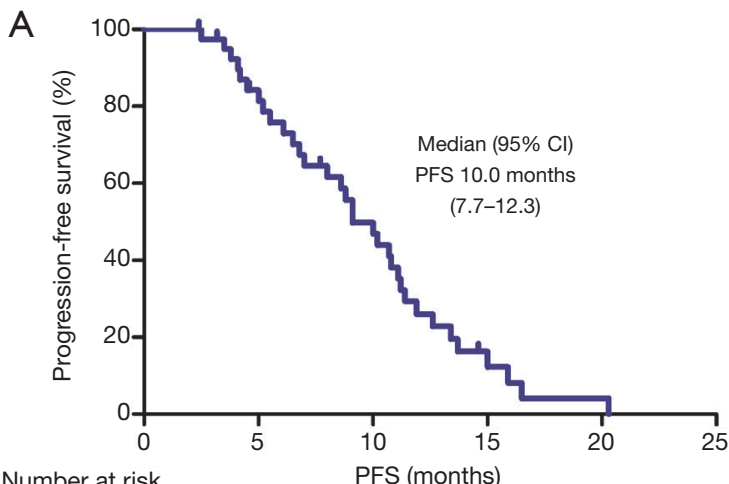

Number at risk

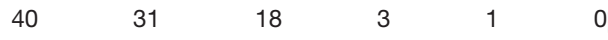

B

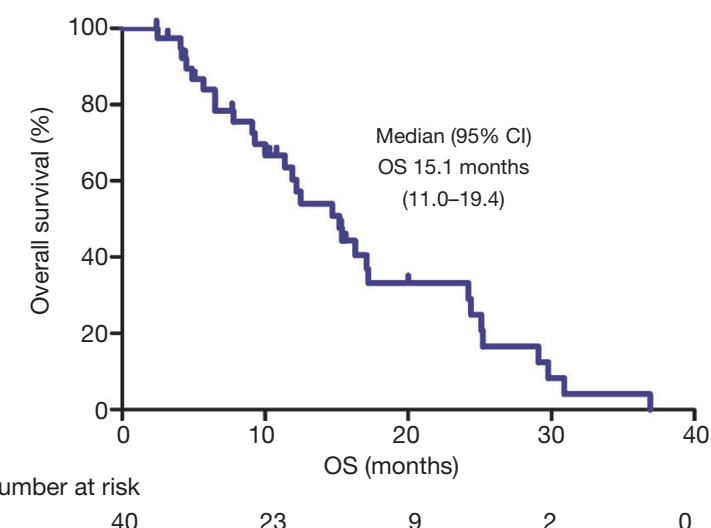

Figure 1 Kaplan-Meier curves of PFS (A) and OS (B) with osimertinib treatment in pretreated EGFRm NSCLC patients with LM. PFS, progression-free survival; OS, overall survival; EGFRm, epidermal growth factor receptor-mutated; NSCLC, non-small cell lung cancer; LM, leptomeningeal metastasis.

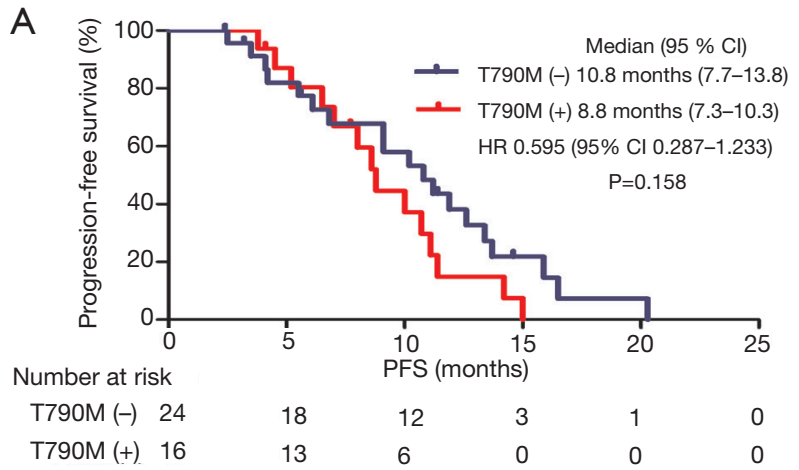

B

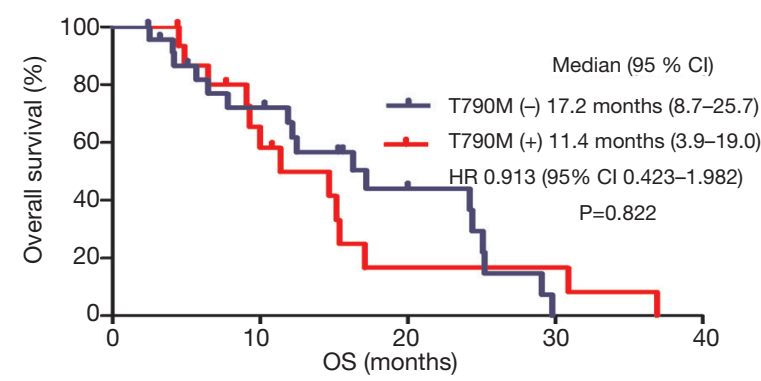

Number at risk

$\begin{array}{llllll}\operatorname{T790M}(-) & 24 & 15 & 7 & 0 & 0 \\ \operatorname{T790M}(+) & 16 & 8 & 2 & 2 & 0\end{array}$

Figure 2 Kaplan-Meier curves of PFS (A) and OS (B) with osimertinib treatment in pretreated EGFRm NSCLC patients with LM according to T790M status. PFS, progression-free survival; OS, overall survival; EGFRm, epidermal growth factor receptormutated; NSCLC, non-small cell lung cancer; LM, leptomeningeal metastasis. 
Table 3 Correlations of T790M detection between samples

\begin{tabular}{lccc}
\hline \multirow{2}{*}{$\begin{array}{l}\text { Cerebrospinal } \\
\text { fluid-plasma }(\mathrm{n}=40)\end{array}$} & \multicolumn{2}{c}{ Cerebrospinal fluid } & Total \\
\cline { 2 - 3 } Plasma & T790M (+) & T790M (-) & \\
T790M (+) & 5 & 8 & 13 \\
T790M (-) & 3 & 24 & 27 \\
Total & 8 & 32 & 40 \\
\hline
\end{tabular}

\section{Correlation between paired CSF and plasma samples and genetic beat map analysis}

Genomic profiles were obtained from the 40 patients with paired CSF and plasma samples. EGFR sensitizing mutations were detected in the CSF (39/40, 97.5\%) and plasma $(20 / 40,50 \%)$ samples, with a concordance rate of $52.5 \%$. T790M mutation is the most common acquired mechanism of resistance in NSCLC patients who receive first-generation EGFR TKIs. The rate of T790M mutation positivity was $40 \%$ ( $\mathrm{n}=16$, including 8 cases in plasma, 3 in CSF, 5 in both CSF and plasma). The consistency between T790M mutation detection in CSF and plasma was $72.5 \%$ (double-positive in 5 pairs and double-negative in 24 pairs) (Table 3). The detection rate of T790M mutations in plasma samples was significantly higher than that in CSF samples $(\mathrm{P}=0.043) . M E T$ amplification is another acquired resistance mechanism to EGFR-TKIs in patients NSCLC. In this study, MET amplification was detected in $7.5 \%(3 / 40)$ of the CSF samples but in none of the plasma samples. The results of analysis also showed that EGFR amplification (10\%), PI3KCA mutation (2.5\%), and PTEN mutation (2.5\%) were frequently detected in the CSF samples (Figure 3). TP53 loss was also identified in circulating tumor DNA (ctDNA) in 19 out of 40 CSF samples, which was higher than the number of paired plasma samples with TP53 loss $(9 / 40, \mathrm{P}=0.002)$.

\section{Discussion}

Given its poor prognosis, leptomeningeal carcinomatosis is selected as an exclusion criterion in the majority of clinical trials for NSCLC. In the present study, osimertinib $80 \mathrm{mg}$ once daily exhibited good efficacy in patients with EGFRm NSCLC who had progressed on first-generation EGFRTKIs and had cytologically confirmed symptomatic LM, regardless of their T790M status. Based on the RANOLM criteria, osimertinib treatment yielded an intracranial

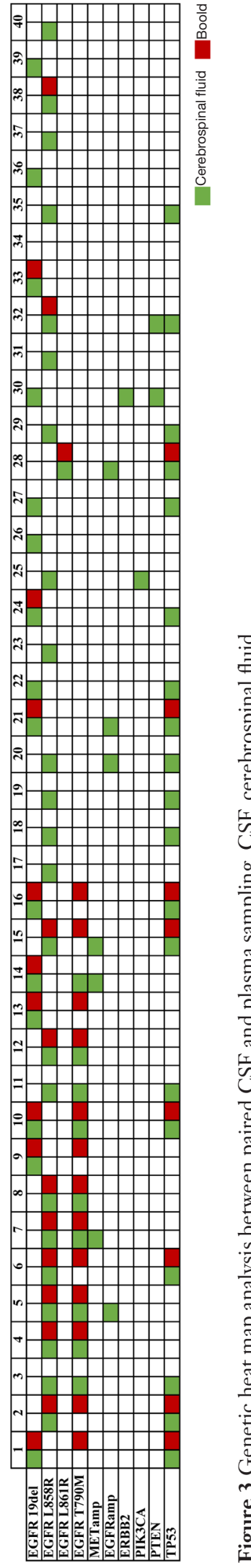


ORR and DCR of $20 \%$ and $95 \%$, respectively, with the median PFS and OS being 10.0 months and 15.1 months, respectively. These results indicate that osimertinib might be an effective option for the treatment of LM.

In the FLAURA study, compared with standard-of-care EGFR-TKIs (erlotinib or gefitinib), osimertinib reduced the risk of intracranial progression by $53 \%$ in the subset of patients with untreated asymptomatic NSCLC, and achieved significantly longer systemic PFS in all predefined subgroups, with a median duration of CNS response of 15.2 months (20). Nanjo et al. (21) verified the efficacy of osimertinib at a standard dose of $80 \mathrm{mg}$ daily in their prospective pilot study of 13 cases with T790M-positive NSCLC for whom erlotinib, gefitinib, or afatinib therapy had failed. They reported the median PFS with osimertinib to be 7.2 months with an improved performance and better neurological findings compared with classic EGFR-TKIs. Ramalingam et al. (22) observed encouraging activity of $160 \mathrm{mg}$ osimertinib in EGFR TKI-pretreated NSCLC patients with T790M-positive LM. An ORR of $27 \%$, a median PFS of 8.6 months and a median OS of 11.0 months were reported. In our study, osimertinib $80 \mathrm{mg}$ was found to have similar efficacy to the $160 \mathrm{mg}$ dose used in the BLOOM study based on the RANO evaluation criteria, even in some patients with a poor performance status. Considering that all patients in the present study received intrathecal injection of methotrexate, with $65 \%$ of them receiving intrathecal injections more than 4 times. In the initial diagnosis of meningeal metastasis, the purpose of intrathecal injection of methotrexate was to decrease intracranial pressure and improve patient's neurological symptoms. In the BLOOM study, the dose of osimertinib $160 \mathrm{mg}$ resulted in discontinuation in $22 \%$ of patients and a dose reduction in $12 \%$ of ones due to aside effects. Compared with the BLOOM study, no patients in our study discontinued osimertinib $80 \mathrm{mg}$ treatment. It could be inferred that the combination standard dose of osimertinib and intrathecal treatment may also improve the clinical outcomes of EGFRm NSCLC patients with LM with less drug reduction and discontinuation.

Previous studies have recommended platinum-based chemotherapy as a second-line therapy for patients with T790M-negative NSCLC (23-25). However, due to the existence of the blood-brain barrier (BBB), which limits the efficacy of chemotherapeutic drugs, as well as a poor ECOG score, platinum-based chemotherapy is not option for most patients with T790M-negative NSCLC in clinical practice. Chalmers et al. (26) reported a long-term response to osimertinib in a patient with positive CSF tumor cells without T790M mutation. Hu et al. (27) also demonstrated that osimertinib may be an effective treatment for patients with LM of NSCLC with EGFR sensitizing mutation without T790M mutation by using NGS detection in puncture tissues and plasma. Interestingly, in our study, osimertinib $80 \mathrm{mg}$ once daily showed a similar efficacy for LM patients with T790M-negative status and those with T790M-positive status. Patients with a T790Mnegative status based on NGS of plasma had prolonged PFS and fewer progression events in comparison with those with a plasma T790M-positive status (28). Although no significant difference was observed in PFS between the T790M-positive and T790M-negative groups in this study, a trend of numerically longer PFS was observed in T790M-negative patients. Therefore, we speculate that osimertinib, with its adequate BBB-penetrating capabilities, can provide good efficacy for LM patients harboring EGFR sensitizing mutations. Moreover, osimertinib may inhibit the emergence of acquired T790M mutation, thus further improving the clinical outcomes of patients with T790Mnegative NSCLC with LM.

Plasma-based liquid biopsy is an extremely common testing method for patients with advanced NSCLC, especially for those who have received prior treatment, due to its high accessibility in clinical practice. However, another important observation of our current research is that ctDNA in plasma may not fully represent the molecular landscape of patients with meningeal metastases, due to the BBB. Our results suggested that plasma and CSF testing should be complementary. In our study, EGFR sensitizing mutations were detected in $97.5 \%$ of CSF samples and $50 \%$ of plasma ones, respectively. A lower frequency of T790M mutation, the most common mechanism for acquired resistance to first-generation EGFR-TKIs, was observed in the CSF samples (20\%) than in the plasma samples (32.5\%), which was consistent with the findings of previous reports that T790M occurrence is more likely at extracranial sites (29). Jiang et al. (30) also demonstrated that NGS uncovered the heterogeneity between CSF and plasma ctDNA among patients with NSCLC with leptomeningeal carcinomatosis. This could be attributed to the fact that the intact BBB inhibits the penetration of first-generation EGFR-TKIs into the CSF, resulting in the control of extracranial disease but intracranial progression. Because this problem is not caused by drug-resistant mutations, no acquired T790M mutation could be detected in the tumor cells in the CSF after progression on first-generation 


\section{Page 8 of 10}

EGFR-TKIs. Another acquired resistance mechanisms included MET amplification (7.5\%), EGFR amplification (10\%), PI3KCA mutation (2.5\%), and PTEN mutation (2.5\%). PTEN is a tumor suppressor gene. PTEN promoter methylation accounted for the decreased expression of PTEN. The sensitivity of drug-resistant cell line to gefitinib and erlotinib was restored after treating with $5 \mathrm{AZA}-\mathrm{CdR}$ induced the expression of PTEN in drug-resistant cell line. Maeda et al. (31) indicated that EGFR-TKI combined with epigenetic modulators can overcome drug resistance. Therefore, epigenetic changes are also an important mechanism of EGFR-TKI acquired resistance.

Several limitations in our study must be acknowledged. First, this was a retrospective study with a limited sample size, and the therapeutic efficacy was assessed by clinicians, which might have introduced potential bias. Thus, the results must be interpreted cautiously. Second, due to a lack of head-to-head comparison between different doses of osimertinib, it remains unclear whether doubledose osimertinib (160 mg once daily) can outperform the standard dose (80 mg once daily) for CNS disease control. Finally, we did not analyze whether the combination of osimertinib and bevacizumab could overcome neurological progression in patients with EGFRm NSCLC due to the irregular use of bevacizumab.

In summary, our data indicate that osimertinib at dose of $80 \mathrm{mg}$ once daily has good efficacy in patients with EGFRm NSCLC with LM for whom prior EGFR-TKI treatment has failed, regardless of their T790M status. In clinical practice, CSF testing is highly recommended, especially for patients with $E G F R m$ NSCLC with symptomatic LM who have previously received EGFR-TKI treatment. The results of this study suggest that the combination of CSF and plasma testing should be complementary. Clinical studies involving larger-scale samples are needed to confirm our observational results.

\section{Acknowledgments}

We would like to take the opportunity to thank the study participants, their families, and everyone who contributed to this research.

Funding: This work was supported by a grant from Beijing Hope Run Special Fund of Cancer Foundation of China (LC2019A15), WUJIEPING Medical Foundation (320.6750.2020-05-4) and the CAMS innovation Fund for Medical Sciences (CIFMS; No. 2019-I2M-2-003).

\section{Footnote}

Reporting Checklist: The authors have completed the STROBE reporting checklist. Available at http://dx.doi. org/10.21037/atm-21-1249

Data Sharing Statement: Available at http://dx.doi. org/10.21037/atm-21-1249

Conflicts of Interest: All authors have completed the ICMJE uniform disclosure form (available at http://dx.doi. org/10.21037/atm-21-1249). Dr. HX reports that this work was supported by a grant from Beijing Hope Run Special Fund of Cancer Foundation of China (LC2019A15), WUJIEPING Medical Foundation (320.6750.2020-05-4). Dr. YW reports that this work was supported by a grant from the CAMS innovation Fund for Medical Sciences (CIFMS; No. 2019-I2M-2-003). The other authors have no conflicts of interest to declare.

Ethical Statement: The authors are accountable for all aspects of the work in ensuring that questions related to the accuracy or integrity of any part of the work are appropriately investigated and resolved. As an observational study without therapeutic intervention, the present study was exempted from obtaining patient informed consent, and the study was approved by the institutional ethics committee of National Cancer/Cancer Hospital, Chinese Academy of Medical Sciences \& Peking Union Medical College (approval 20-114/2310). The study was conducted in accordance with the Declaration of Helsinki (as revised in 2013).

Open Access Statement: This is an Open Access article distributed in accordance with the Creative Commons Attribution-NonCommercial-NoDerivs 4.0 International License (CC BY-NC-ND 4.0), which permits the noncommercial replication and distribution of the article with the strict proviso that no changes or edits are made and the original work is properly cited (including links to both the formal publication through the relevant DOI and the license). See: https://creativecommons.org/licenses/by-nc-nd/4.0/.

\section{References}

1. Rangachari D, Yamaguchi N, VanderLaan PA, et al. Brain metastases in patients with EGFR-mutated or ALKrearranged non-small-cell lung cancers. Lung Cancer 
2015;88:108-11.

2. Li YS, Jiang BY, Yang JJ, et al. Leptomeningeal metastases in patients with NSCLC with EGFR mutations. J Thorac Oncol 2016;11:1962-9.

3. Kuiper JL, Hendriks LE, van der Wekken AJ. et al. Treatment and survival of patients with EGFRmutated non-small cell lung cancer and leptomeningeal metastasis: a retrospective cohort analysis. Lung Cancer 2015;89:255-61.

4. Riess JW, Nagpal S, Iv M, et al. Prolonged survival nonsmall-cell lung cancer with leptomeningeal carcinomatosis in the modern treatment era. Clin Lung Cancer 2014;15:202-6.

5. Cheng H, Perez-Soler R. Leptomeningeal metastases in non-small-cell lung cancer. Lancet Oncol 2018;19:e43-55.

6. Morris PG, Reiner AS, Szenberg OR, et al. Leptomeningeal metastasis from non-small lung cancer: survival and the impact of whole brain radiotherapy. J Thorac Oncol 2012;7:382-5.

7. Wu YL, Zhou L, Lu Y, et al. Intrathecal chemotherapy as a treatment for leptomeningeal metastasis of nonsmall cell lung cancer: A pooled analysis. Oncol Lett 2016;12:1301-14.

8. Mok TS, Wu YL, Thongprasert S, et al. Gefitinib or carboplatin-paclitaxel in pulmonary adenocarcinoma. $\mathrm{N}$ Engl J Med 2009;361:947-57.

9. Chen G, Feng J, Zhou C et al. Quality of life (QoL) analyses from OPTIMAL (CTONG-0802), a phase III, randonmised, open-label study of first-line erlotinib versus chemotherapy in patients with advanced EGFR mutationpositive non-small-cell lung cancer (NSCLC). Ann Oncol 2013;24:1615-22.

10. Shi YK, Wang L, Han BH et al. First-line icotinib versus cisplatin/pemetrexed plus pemetrexed maintenance therapy for patients with advanced EGFR mutation-positive lung adenocarcinoma (CONVINCE): a phase 3, open-label, randomized study. Ann Oncol 2017;28:2443-50.

11. Sequist LV, Yang JC, Yamamoto N, et al. Phase III study of afatinib or cisplatin plus pemetrexed in patients with metastatic lung adenocarcinoma with EGFR mutations. J Clin Oncol 2013;31:3327-34.

12. Mok TS, Cheng Y, Zhou X, et al. Improvement in overall survival in a randomized study that compared dacomitinib with gefitinib in patients with advanced non-small cell lung cancer and EGFR-activating mutations. J Clin Oncol 2018;36:2244-50.

13. Soria JC, Ohe Y, Vansteenkiste J, et al. Osimertinib in untreated EGFR-mutated advanced non-small-cell lung cancer. N Engl J Med 2018;378:113-25.

14. Rosell R, Carcereny E, Gervais R, et al. Erlotinib versus standard chemotherapy as first-line treatment for European patients with advanced EGFR mutation-positive non-small- cell lung cancer (EURTAC): a multicentre open-label randomized phase 3 trail. Lancet Oncol 2012;13:239-46.

15. Schuler M, Wu YL, Hirsh V, et al. First-line Afatinib verus chemotherapy in patients with non-small cell lung cancer and common epidermal growth factor receptor gene mutations and brain metastases. J Thorac Oncol 2016;11:380-90.

16. Wu YL, Ahn MJ, Garassino MC, et al. CNS efficacy of osimertinib in patients with T790M-positive advanced non-small cell lung cancer: data from a randomized phase trail (AURA3). J Clin Oncol 2018;36:2702-9.

17. Yang JCH, Kim SW, Kim DW, et al. Osimertinib in patients with epidermal growth factor receptor mutationpositive non-small-cell lung cancer and leptomeningeal metastases: The BLOOM study. J Clin Oncol 2020;38:538-47.

18. Chamberlain M, Junck L, Brandsma D, et al. Leptomeningeal metastases: A RANO proposal for response criteria. Neuro Oncol 2017;19:484-92.

19. Eisenhauer EA, Therasse P, Bogaerts J, et al. New response evaluation criteria in solid tumors: revised RECIST guideline (version1.1). Eur J Cancer 2009;45:228-47.

20. Reungwetwattana T, Nakagawa K, Cho BC, et al. CNS response to osimertinib versus standard epidermal growth factor receptor tyrosine kinase inhibitors in patients with untreated EGFR-mutated advanced non-small-cell lung cancer. J Clin Oncol 2018;36:JCO2018783118.

21. Nanjo S, Hata A, Okuda C, et al. Standard-dose osimertinib for refractory leptomeningeal metastases in T790M-positive EGFR-mutant non-small cell lung cancer. Br J Cancer 2018;118:32-7.

22. Ramalingam SS, Vansteenkiste J, Planchard D, et al. Overall survival with osimertinib in untreated, EGFR-mutated advanced NSCLC. N Engl J Med 2020;382:41-50.

23. Wu YL, Planchard D, Lu S, et al. Pan-Asian adapted clinical practice guidelines for the management of patients with metastatic non-small cell lung cancer: a CSCOESMO initiative endorsed by JSMO, KSMO, MOS, SSO and TOS. Ann Oncol 2019;30:171-210.

24. Hanna N, Johnson D, Temin S, et al. Systemic therapy for stage IV non-small-cell lung cancer: American Society of Clinical Oncology clinical practice guideline update. J Clin 
Oncol 2017;35:3484-515.

25. Planchard D, Popat S, Kerr K, et al. Metastatic nonsmall cell lung cancer: ESMO clinical practice guideline for diagnosis, treatment and follow-up. Ann Oncol 2018;29:iv192-237.

26. Chalmers A, Jensen L, Akerley W. Durable response to osimertinib in EGFR mutated T790M wild type non-small cell lung cancer with leptomeningeal metastases: a case report. Lung Cancer 2017;114:68-9.

27. Hu X, Chen W, Li X, et al. Clinical efficacy analysis of osimertinib treatment for a patient with leptomeningeal metastasis of EGFR+ non-small cell lung cancer without the T790M mutation. Ann Palliat Med 2019;8:525-31.

28. Papadimitrakopoulou VA, Han JY, Ahn MJ, et al. Epidermal growth factor receptor mutation analysis in tissue and plasma from the AURA3 trial: Osimertinib versus platinum-pemetrexed for T790M mutation-

Cite this article as: $\mathrm{Xu} \mathrm{H}$, Chen H, Kong J, Zhang Y, Liu S, Yang G, Yang L, Wang Y. Osimertinib for the treatment of epidermal growth factor receptor-mutated non-small cell lung cancer patients with leptomeningeal metastases and different T790M status. Ann Transl Med 2021;9(11):937. doi: 10.21037/ atm-21-1249 positive advanced non-small cell lung cancer. Cancer 2020;126:373-80.

29. Nanjo S, Arai S, Wang W, et al. MET copy number gain is associated with gefitinib resistance in leptomeningeal carcinomatosis of EGFR-mutant lung cancer. Mol Cancer Ther 2017;16:506-15.

30. Jiang BY, Li Y, Chuai S, et al. NGS to reveal heterogeneity between cerebrospinal fluid and plasma ctDNA among non-small cell lung cancer patients with leptomeningeal carcinomatosis. Proc Am Soc Clin Oncol 2017;35:9022.

31. Maeda M, Murakami Y,Watari, K., et al. CpG hypermethylation contributes to decreased expression of PTEN during acquired resistance to gefitinib in human lung cancer cell lines. Lung Cancer 2015;87:265-71.

(English Language Editor: J. Reynolds) 


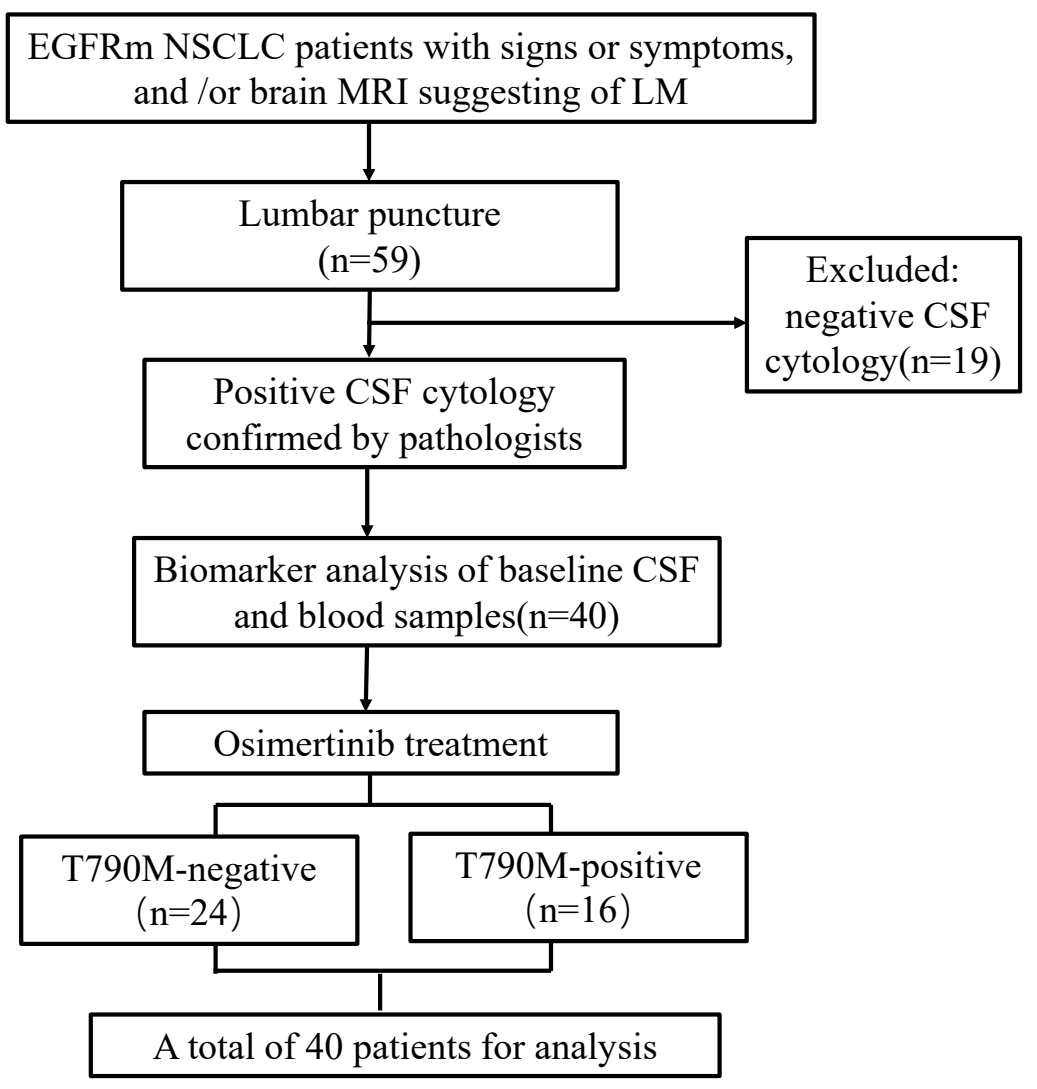

Figure S1 Study flow chart.

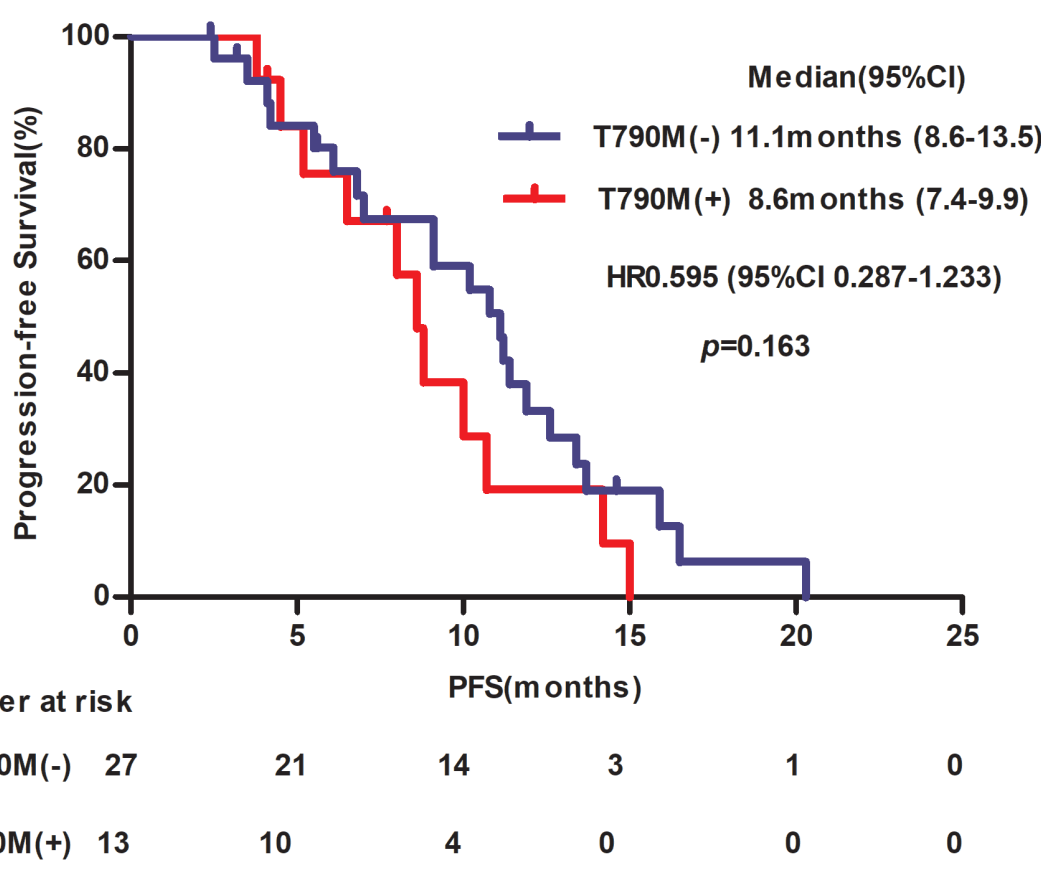

$\operatorname{T790M(+)~} 13$

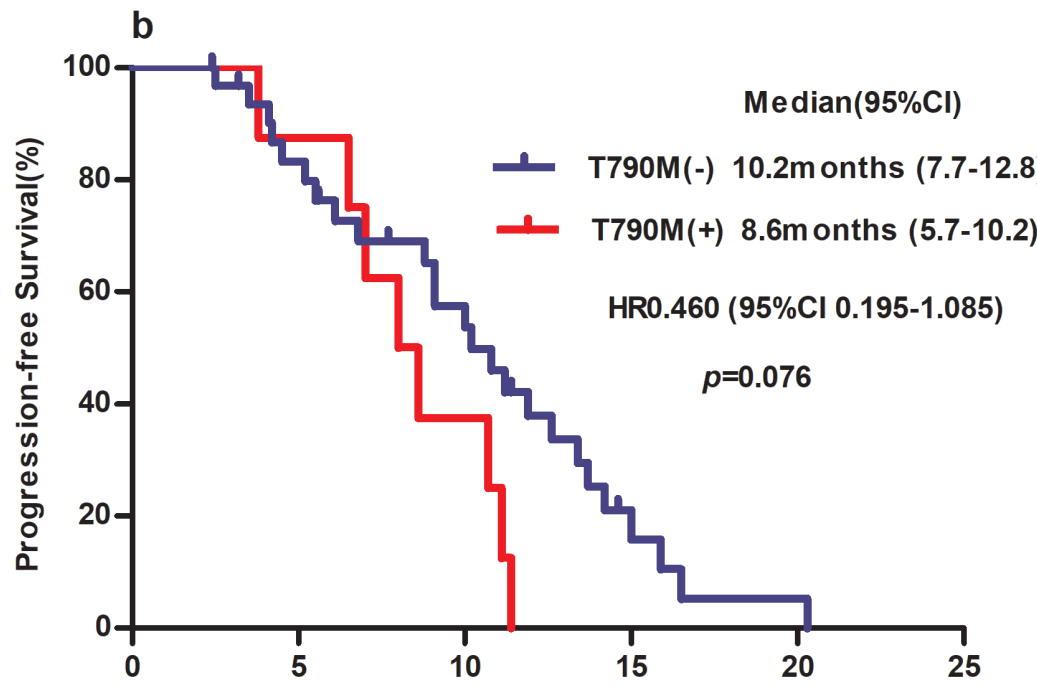

Num ber at risk

PFS(months)

$\begin{array}{lcccccc}\operatorname{T790M}(-) & 32 & 24 & 15 & 3 & 1 & 0 \\ \operatorname{T790M(+)} & 8 & 7 & 3 & 0 & 0 & 0\end{array}$

Figure S2 Kaplan-Meier curves of PFS with osimertinib treatment in plasma and CSF-based on T790M status (2A) in plasma (2B) in CSF. PFS, progression-free survival; CSF, cerebrospinal fluid. 